\title{
Pulsed Field-Induced Magnetization Switching in Antiferromagnetic Ferrihydrite Nanoparticles
}

\author{
D. A. Balaev ${ }^{a, b}, *$, A. A. Krasikov ${ }^{a}$, D. A. Velikanov ${ }^{a}$, S. I. Popkov ${ }^{a, b}$, N. V. Dubynin ${ }^{c}$, \\ S. V. Stolyar ${ }^{a, b, d}$, V. P. Ladygina ${ }^{d}$, and R. N. Yaroslavtsev ${ }^{a, b}$ \\ ${ }^{a}$ Kirensky Institute of Physics, Krasnoyarsk Scientific Center, Siberian Branch, Russian Academy of Sciences, \\ Krasnoyarsk, 660036 Russia \\ ${ }^{b}$ Siberian Federal University, Krasnoyarsk, 660041 Russia \\ ${ }^{c}$ Moscow State University of Civil Engineering, Moscow, 129337 Russia \\ ${ }^{d}$ Krasnoyarsk Scientific Center, Siberian Branch, Russian Academy of Sciences, Krasnoyarsk, 660036 Russia \\ *e-mail: dabalaev@iph.krasn.ru \\ Received March 13, 2018
}

\begin{abstract}
The dynamic magnetization switching of ferrihydrite nanoparticles has been investigated by a pulsed magnetometer technique in maximum fields $H_{\max }$ of up to $130 \mathrm{kOe}$ with pulse lengths of 4,8 , and $16 \mathrm{~ms}$. Ferrihydrite exhibits antiferromagnetic ordering and defects cause the uncompensated magnetic moment in nanoparticles; therefore, the behavior typical of magnetic nanoparticles is observed. The dynamic hysteresis loops measured under the above-mentioned conditions show that the use of pulsed fields significantly broadens the temperature region of existence of the magnetic hysteresis and the coercivity can be governed by varying the maximum field and pulse length. This behavior is resulted from the relaxation effects typical of conventional ferro- and ferrimagnetic nanoparticles and the features typical of antiferromagnetic nanoparticles.
\end{abstract}

DOI: $10.1134 / \mathrm{S} 1063783418100025$

\section{INTRODUCTION}

Nanoparticles of materials characterized by antiferromagnetic (AFM) ordering exhibit the intriguing magnetic properties [1-3]. This is due to the surface effects and defects (AFM ordering violations). The formation of defects on the surface and in the bulk of particles is related to their crystallochemical properties. The defects lead to the occurrence of an uncompensated magnetic moment in AFM nanoparticles, which can attain hundreds of Bohr magnetons. This offers the opportunity of using AFM nanoparticles in various research directions, including medicine $[4,5]$.

Along with the existence of uncompensated magnetic moment $\mu_{p}$, there is a number of other interesting peculiarities in the magnetic behavior of AFM nanoparticles. Similar to ferro- and ferrimagnetic nanoparticles, AFM particles exhibit a superparamagnetic (SP) behavior at temperatures above the characteristic blocking temperature $T_{B}$. In the temperature range of $T<T_{B}$, the $M(H)$ magnetization curves are hysteretic. On the one hand, similar to ferro- and ferrimagnetic nanoparticles, the $M(H)$ hysteresis is related to the competition between the Zeeman energy $\mu_{p} H$ and the magnetic anisotropy energy $K_{\text {eff }} V\left(K_{\text {eff }}\right.$ is the effective magnetic anisotropy constant, which includes the surface anisotropy, and $V$ is the particle volume). On the other hand, the magnetic moment of an AFM particle can be exchange-coupled with the AFM core, which can give rise to an additional anisotropy. This mechanism possibly manifests itself in the shift of the magnetic hysteresis loop of AFM particles after their cooling in external field from a temperature over $T_{B}$ [6-13]. In addition, it is worth noting that the magnetization curve of an ensemble of AF nanoparticles is, in the first approximation (with disregard of the exchange coupling between $\mu_{p}$ and the AFM core), a superposition of the contributions of the magnetic moments of particles and the characteristic field-linear magnetic response of the antiferromagnetically ordered core $[1,2,14-21]$.

In the conventional investigations of the magnetic hysteresis in quasistatic magnetic fields, the external field variation rate $d H / d t$ is usually no higher than $\sim 10^{2} \mathrm{Oe} / \mathrm{s}$. However, study of the dynamical magnetization switching with significantly higher $d H / d t$ values can bring new information, since the variation in the parameter $d H / d t$ affects the relaxation processes [22$24]$. The ratio between the $T_{B}, V$, and $K_{\text {eff }}$ values and characteristic measurement $\left(\tau_{m}\right)$ and particle relax- 
ation $\left(\tau_{0}\right)$ times are determined from the Néel-Brown expression

$$
T_{B}=K_{\mathrm{eff}} V / \ln \left(\tau_{m} / \tau_{0}\right) k_{\mathrm{B}},
$$

where $k_{\mathrm{B}}$ is the Boltzmann constant. The $\tau_{0}$ value can lie between $10^{-9}-10^{-12} \mathrm{~s}$ and the $\tau_{m}$ value in the quasistatic measurements is about $10^{1}-10^{2} \mathrm{~s}$ [1]. The increase in the parameter $d H / d t$ during magnetization is analogous to a decrease in the characteristic time $\tau_{m}$, which results in the effective growth of the SP blocking temperature and the $M(H)$ dependence reversible in the quasistatic measurements can exhibit the hysteresis under the dynamic magnetization switching.

If the field $H_{R}$ of the onset of reversible behavior of the $M(H)$ dependence, (i.e., at $H \geq H_{R}$ the hysteresis loop is closed) is fairly weak (e.g., about $10^{3} \mathrm{Oe}$ ), then the above-mentioned processes can be observed on facilities generating ac magnetic fields of different frequencies. However, if the $H_{R}$ value is $\sim 10^{4}$ Oe or more, then the processes of dynamic magnetization switching can be investigated on facilities generating pulsed magnetic fields. Antiferromagnetic nanoparticles belong to the class of objects with the high $H_{R}$ values $[13,17,25,26]$. The behavior of coercivity $H_{C}$ can only be examined on magnetometers operating in strong pulsed magnetic fields.

In our previous work [27], we studied the dynamic magnetization switching of $\varepsilon-\mathrm{Fe}_{2} \mathrm{O}_{3}$ nanoparticles in pulsed magnetic fields. The $H_{R}$ value of these objects is $\sim 50 \mathrm{kOe}$. Using the dynamic magnetic hysteresis loops, we obtained the coercivity as a function of the external field variation rate $d H / d t$, which allowed us to establish the role of surface anisotropy in these objects using a theoretical model of ferromagnetic nanoparticles $[22,23]$. In our opinion, it is reasonable to extend these dynamic magnetization switching investigations to AFM nanoparticles. The aim of this study was to establish the regularities in the behavior of dynamic magnetic hysteresis (DMH) loops of antiferromagnetically ordered ferrihydrite.

Ferrihydrite is an iron hydroxide with the nominal formula $5 \mathrm{Fe}_{2} \mathrm{O}_{3} \cdot 9 \mathrm{H}_{2} \mathrm{O}$, which exists in a nanosized form. Due to the surface and bulk defects, particles 3$5 \mathrm{~nm}$ in size have an uncompensated magnetic moment of about $150-300 \mu_{\mathrm{B}}$ in $[6,14-16,26,28-31]$. In addition, ferrihydrite is a part of the biological object ferritin contained in living organisms. Pure ferrihydrite can be obtained chemically or by extracting from the products of vital activity of bacteria cultivated under certain conditions (biogenic ferrihydrite).

The investigations were carried out on the ferrihydrite samples of both types.

\section{EXPERIMENTAL}

\subsection{Fabrication and Characterization of the Ferrihydrite Samples}

The technique for preparing biogenic ferrihydrite formed by the Klebsiella oxytoca bacteria vital activity was described in detail in [31, 32]. Chemical ferrihydrite was obtained by slow adding the $\mathrm{NaOH}(1 \mathrm{M})$ alkali solution to the $\mathrm{FeCl}_{3}$ iron chloride solution $(0.02 \mathrm{M})$ upon constant stirring at room temperature until obtaining the neutral $\mathrm{pH}$ value [33]. The prepared samples were annealed in air at $170^{\circ} \mathrm{C}$ for $24 \mathrm{~h}$. These samples are hereinafter referred to as Bio-FH and Chem-FH.

Analysis of the Mössbauer spectra obtained on an MC-1104Em spectrometer $\left({ }^{57} \mathrm{Co}(\mathrm{Cr})\right.$ source $)$ showed that the data for the obtained samples are in good agreement with the results reported in [31-33] and the annealing did not lead to the occurrence of foreign iron oxide phases. According to the transmission electron microscopy data, the average Bio-FH and ChemFH particle size $\langle d\rangle$ was $\sim 4$ and $\sim 5 \mathrm{~nm}$, respectively.

The static magnetization measurements $(M(T)$ dependences) were performed on a SQUID magnetometer [34].

\subsection{Measuring the Dynamic Magnetic Hysteresis Loops}

The DMH loops were measured using an induction magnetometer in pulsed magnetic fields induced by a standard method of capacitor bank discharging through a solenoid. The possibility of measuring the hysteresis loops was ensured by the periodic operation of a facility generating pulsed magnetic fields. In this case, the external field was increased to $H_{\max }$ (in these experiments, up to $130 \mathrm{kOe}$ ), then to a negative value somewhat lower than $\left|H_{\text {max }}\right|$, and, after that, to zero (via closing a thyristor unit). The pulse length was varied by switching the capacitor banks to different capacitances. The measurements were performed at pulse lengths $\tau_{P}\left(\tau_{P}\right.$ is the half-period during which the external field changes from $H=0$ to $H_{\max }$ and then to $H=0$ ) of 4,8 , and $16 \mathrm{~ms}$, which, together with the change in the maximum field strength $H_{\max }$ in the pulse makes it possible to measure the $\mathrm{DMH}$ in a wide range of field variation rates $d H / d t \sim 10-80 \mathrm{MOe} / \mathrm{s}$. The measurements were performed at a temperature of $77 \mathrm{~K}$. The magnetic field variation rate at the instant of sample magnetization switching was determined from the near-zero $d H / d t$ value using the experimental $H(t)$ dependences.

\section{RESULTS AND DISCUSSION}

Figure 1 shows temperature dependences of magnetization $M(T)$ for the investigated samples, which were measured under zero-field cooling conditions 


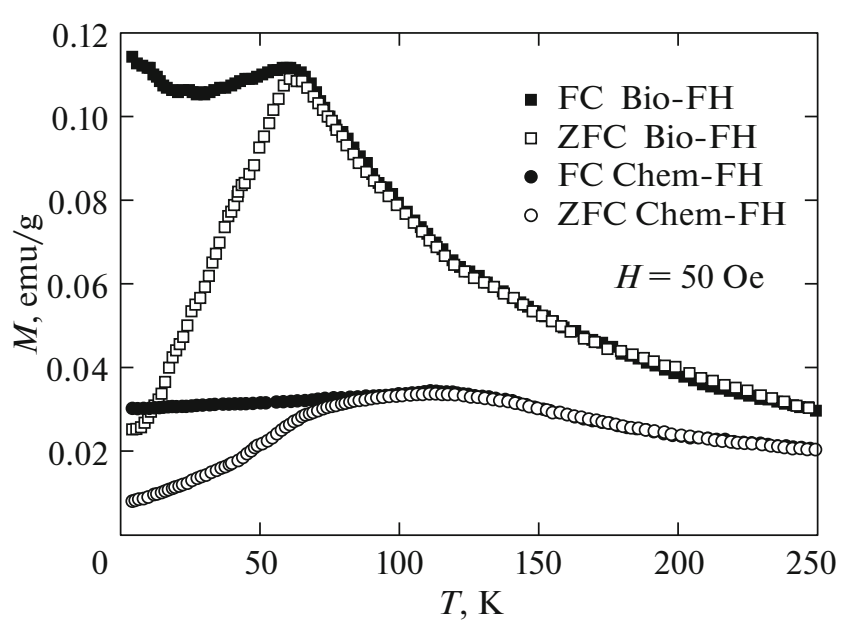

Fig. 1. ZFC and FC temperature dependences of magnetization $M(T)$ for the nanoferrihydrite samples.

(ZFC) and upon cooling in an external field of $H=$ 50 Oe (FC). The shape of these dependences shows that the samples under study exhibit a characteristic SP behavior: the pronounced $M(T)_{\mathrm{ZFC}}$ maximum and a discrepancy between the $M(T)_{\mathrm{ZFC}}$ and $M(T)_{\mathrm{FC}}$ dependences. If the SP blocking temperature $T_{B}$ is determined at the maximum point of the $M(T)_{\mathrm{ZFC}}$ dependence, then, the $T_{B}$ values are 61 and $111 \mathrm{~K}$ for the Bio-FH and Chem-FH samples, respectively. At $T \leq T_{B}$, the discrepancy between the $M(T)_{\mathrm{ZFC}}$ and $M(T)_{\mathrm{FC}}$ dependences is observed. The $T_{B}$ values are noticeably higher than those for the initial (unannealed) samples $(\approx 23$ and $44 \mathrm{~K})$ and, as was shown previously $[31,35,36]$, the increase in the $T_{B}$ values is related to an increase in the particle size upon lowtemperature annealing.

At a temperature of $T=77 \mathrm{~K}$, the magnetic moments of Bio-FH particles are in the SP state under the quasistatic magnetic measurement conditions. In these measurements, the $M(H)$ dependences are completely reversible $\left(H_{C}=0\right)$. The $T_{B}$ value for the Chem-FH sample is somewhat higher than $77 \mathrm{~K}$. However, according to our data, in the quasistatic magnetic measurements (up to $60 \mathrm{kOe}$ ), the $H_{C}$ value at this temperature is no higher than $10^{2} \mathrm{Oe}$.

Figure 2 shows DMH loops for the investigated samples. For the abscissa axis scale used ( $\pm 155 \mathrm{kOe})$, the $M(H)$ data for all the maximum applied field $H_{\text {max }}$ values and pulse lengths $\tau_{P}$ lie within the line thickness. The insets in Fig. 2 illustrate the behavior of DMH loops at the origin of coordinates. It can be seen that the $M(H)$ dependences are characterized by the coercivity $H_{C}$. The $H_{C}$ value for DMH loops is determined as an absolute value of the abscissa of the point of intersection of the $M(H)$ dependence with the $H$ axis in the range of $H<0$. The $H_{C}$ value depends on the
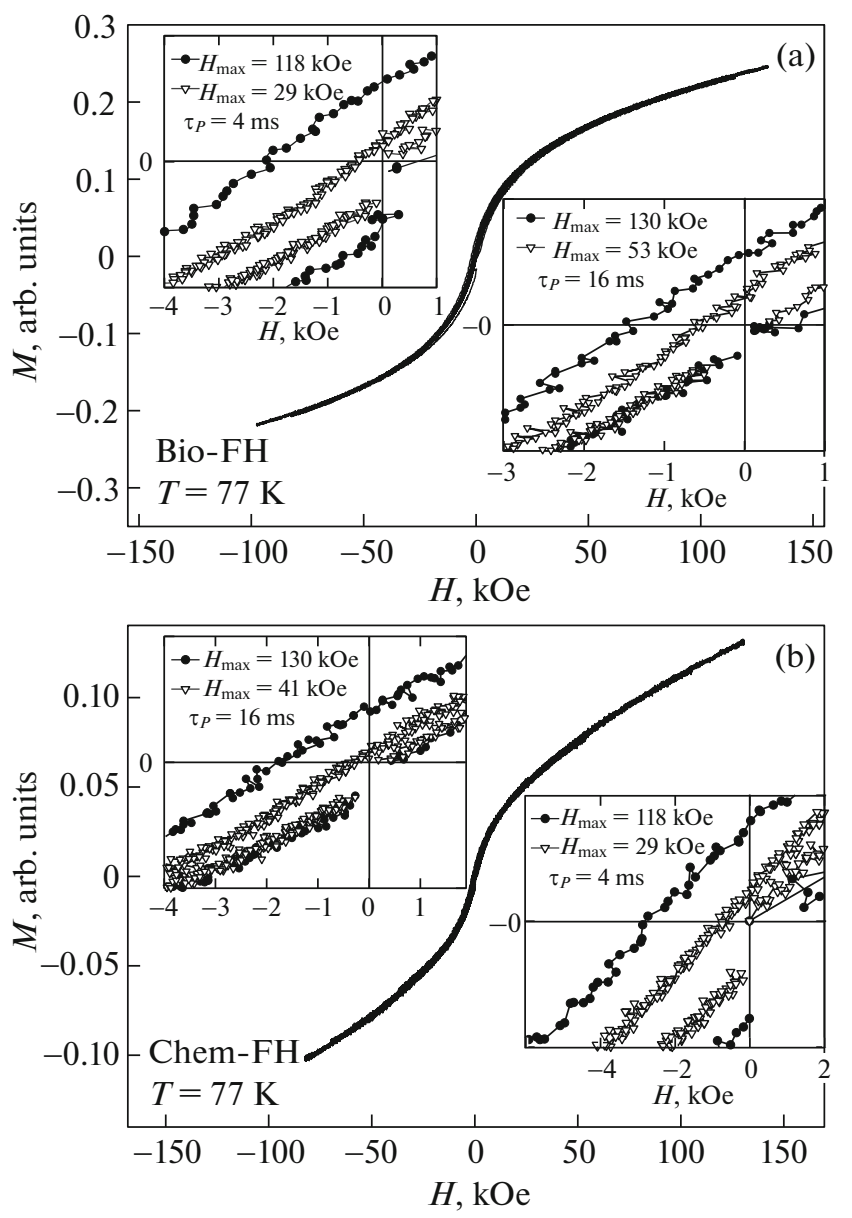

Fig. 2. $M(H)$ dependences under pulsed magnetization switching of (a) biogenic and (b) chemical nanoferrhydrite at $T=77 \mathrm{~K}$. Inserts: course of the $M(H)$ dependences at the origin of coordinates at different maximum fields $H_{\max }$ and pulse lengths $\tau_{P}$. Note that under pulsed magnetization switching, the variation in the external field $H$ follows the cycle $0 \rightarrow H_{\max } \rightarrow-H_{\max } \rightarrow 0$.

pulse length and $H_{\max }$ value. The broadening of the region of hysteresis existence under the pulsed magnetization switching can be easily understood by considering the experimental conditions using Eq. (1). Usually, if we speak about the temperature measurements of magnetic susceptibility for the quasistatic measurements, the $\tau_{m}$ value is taken to be $10^{1}-10^{2} \mathrm{~s}$ [1]; if we assume $\tau_{m}$ to be the hysteresis loop measurement time, then, at a typical value of $d H / d t \sim 10^{1}-10^{2} \mathrm{Oe}$, the $\tau_{m}$ value will be $\sim 10^{4} \mathrm{~s}$. For the case of ac fields with frequency $\omega$, we obviously have $\tau_{m}=2 \pi / \omega$. If we use this approach in the pulsed measurements, for which $\omega=$ $\pi / \tau_{P}$ (see Subsection 2.2), then we have $\tau_{m}=2 \tau_{P}$. Thus, at the shortest pulse length $\tau_{P}=4 \mathrm{~ms}$, we obtain at $\tau_{0} \sim 10^{-10}-10^{-11} \mathrm{~s}$ that the $T_{B}$ value increases approximately by a factor of $1.5-1.7$ as compared with the quasistatic conditions. This leads to the fact that 

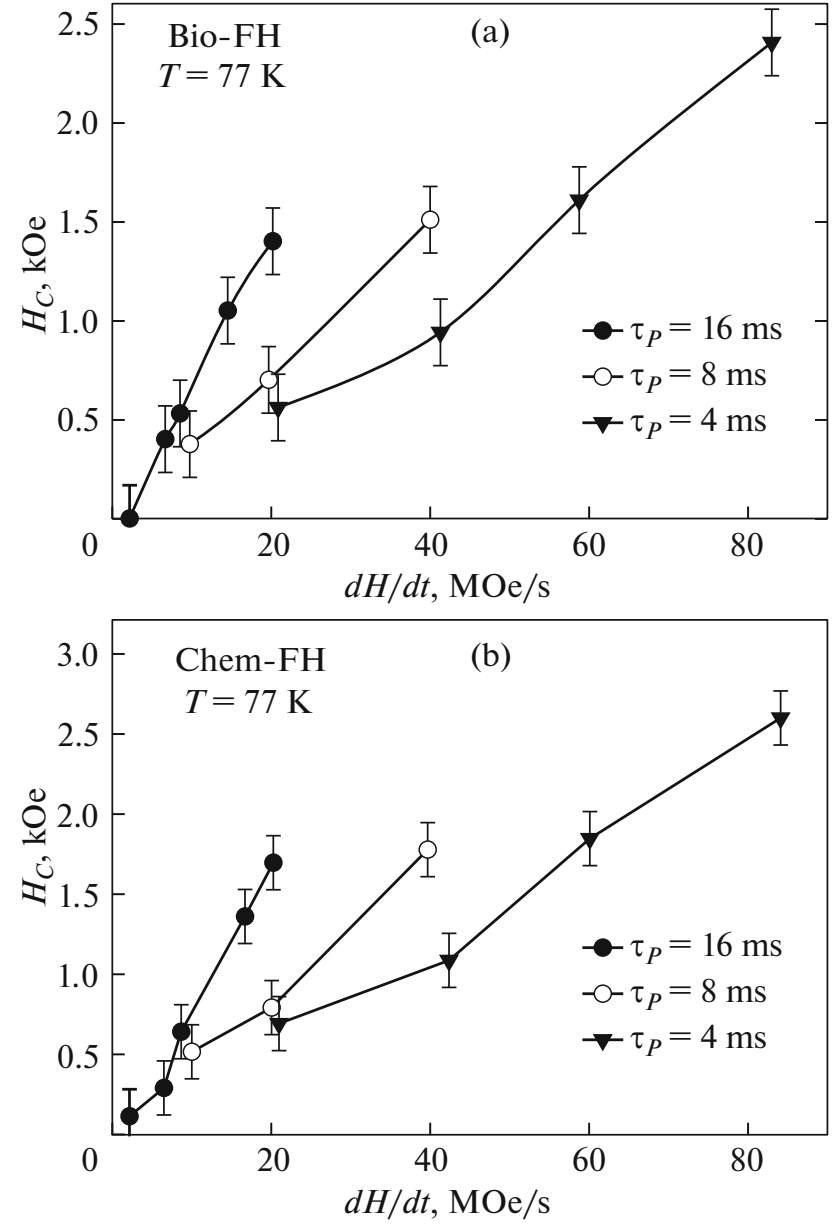

Fig. 3. Dependences of the coercivity $H_{C}$ under pulsed field-induced magnetization switching as a function of the field variation rate $d H / d t$ for (a) the biogenic and (b) chemical nanoferrihydrite samples at $T=77 \mathrm{~K}$. The data are grouped (symbols and lines) by pulse lengths $\tau_{P}$.

the effective blocking temperature increases and the measuring temperature $77 \mathrm{~K}$ hits the range where the particle magnetic moments are blocked. In principle, the observed coercivity growth with the ac field frequency $\left(\omega=\pi / \tau_{P}\right)$ does not contradict the theoretical results for AFM nanoparticles from [24]. However, at this stage of investigations, it is difficult to make a comparison with the theory.

Figure 3 shows the dependence of $H_{C}$ on the field variation rate $d H / d t$ for the investigated samples. The data in Fig. 3 are grouped (symbols and lines) by pulse lengths $\tau_{P}$. It can be seen that, despite the general trend of the $H_{C}$ growth with $d H / d t$, the bright $H_{C}(d H / d t)$ dependence is not observed. This behavior differs from the data obtained previously for $\varepsilon-\mathrm{Fe}_{2} \mathrm{O}_{3}$ nanoparticles [27], which can be considered as singledomain ferro- or ferrimagnetic nanoparticles as applied to the magnetization process [37, 38]. The investigated ferrihydrite samples exhibit AFM order-
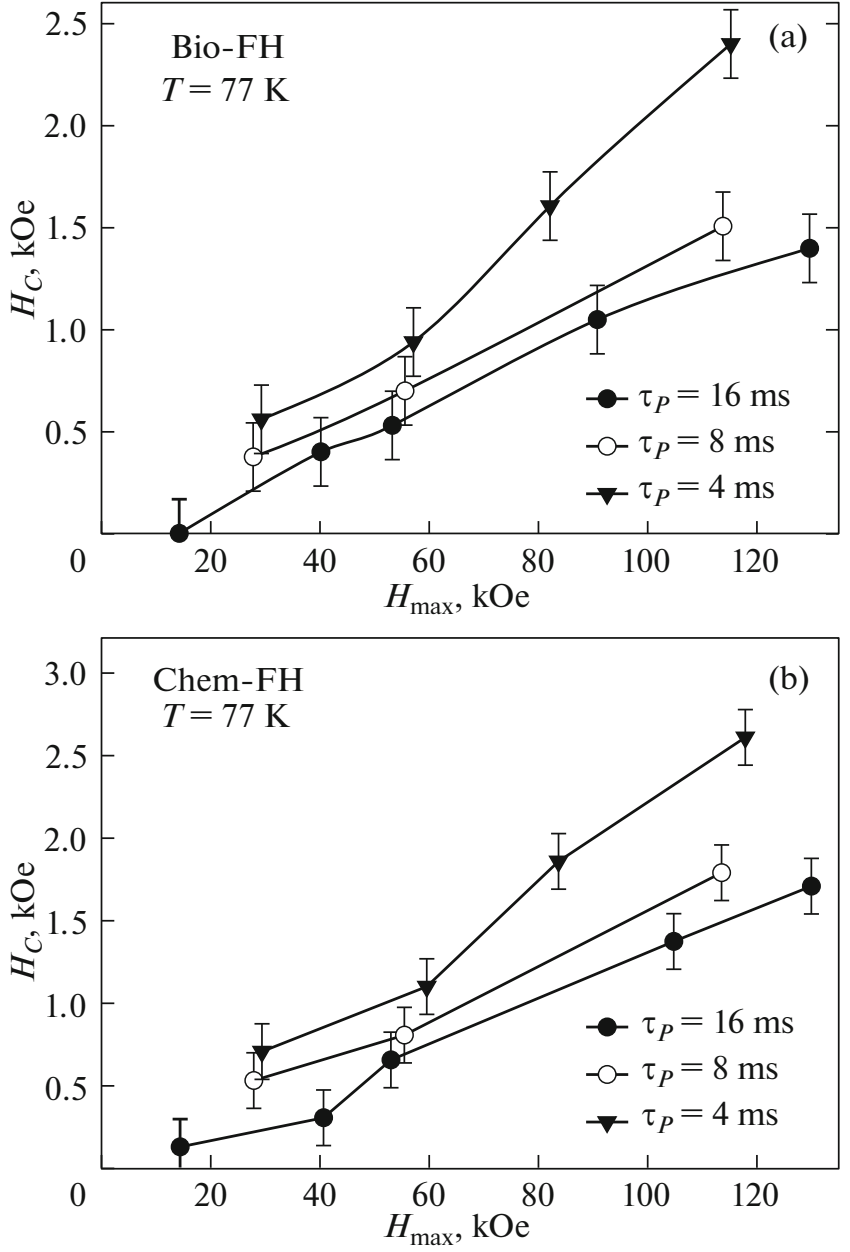

Fig. 4. Dependences of the coercivity $H_{C}$ under pulsed field-induced magnetization switching as a function of the maximum applied field $H_{\max }$ for (a) the biogenic and (b) chemical nanoferrihydrite samples at $T=77 \mathrm{~K}$. The data are grouped (symbols and lines) by pulse lengths $\tau_{P}$.

ing; for this kind of particles, there is apparently an additional factor responsible for the coercivity in the DMH measurements. In our opinion, this factor can be the maximum applied field. Indeed, according to the results of investigation of nanoferrihydrites in quasistatic magnetic fields, these objects are characterized by the strong fields of irreversible behavior of magnetization $H_{R}[9,13]$, which can attain hundreds of kilooersted at low temperatures. As a result, the $H_{C}$ value depends on the $H_{\max }$ value.

In view of the aforesaid, the coercivity data obtained here are built as a function of the maximum applied field $H_{\max }$ (see Fig. 4). Similar to the previous figure, the data are grouped (symbols and connecting lines) by pulse lengths $\tau_{P}$. It can be seen that the $H_{C}\left(H_{\max }\right)$ dependences at $\tau_{P}=$ const are ascending and the effect of pulse length on the $H_{C}$ value is clearly observed. As was found in $[9,12,13]$, at the quasistatic 
measurements, the $H_{C}\left(H_{\max }\right)$ dependences for nanoferrihydrite are $S$-shaped. In principle, despite the data spread in Fig. 4, we may conclude that the $H_{C}\left(H_{\max }\right)$ dependences in the field range of $H_{\max }>$ $80 \mathrm{kOe}$ have a negative curvature, i.e., tend to saturation, which agrees qualitatively with the results reported in $[9,12,13]$. Hence, the maximum applied field affects strongly the DMH behavior in nanoferrihydrite.

On the other hand, if we fix the $H_{\max }$ value for the data in Fig. 4 and consider the $H_{C}\left(\tau_{P}\right)$ dependences (cross section at $H_{\max } \approx$ const), then we will see that the coercivity obviously increases with decreasing pulse length: $H_{C}\left(\tau_{P}=4 \mathrm{~ms}\right)>H_{C}\left(\tau_{P}=8 \mathrm{~ms}\right)>H_{C}\left(\tau_{P}=\right.$ $16 \mathrm{~ms})$ at $H_{\max } \approx$ const. This behavior can be considered to be the manifestation of the effect of dynamic magnetization switching on the coercivity of the investigated samples.

We may point out roughly that under the dynamic magnetization switching, an increase in the $H_{\max }$ value leads to an increase in $H_{C}$ by approximately the same value as the decrease in the pulse length from 16 to $4 \mathrm{~ms}$. For example, according to the data in Fig. 4, an increase in the $H_{\max }$ value to $\sim 100 \mathrm{kOe}$ leads to an increase in $H_{C}$ of up to $1.3-1.5 \mathrm{kOe}$ at $\tau_{P}=16 \mathrm{~ms}$ (taking into account that the $H_{C}$ value in the quasistatic magnetic measurements is no more than $10^{2} \mathrm{Oe}$ ). At $H_{\max } \sim 120 \mathrm{kOe}$, a decrease in $\tau_{P}$ from 16 to 4 ms leads to an additional increase in $H_{C}$ to $2.4-2.5 \mathrm{kOe}$.

\section{CONCLUSIONS}

Thus, we experimentally investigated the dynamic magnetization switching of antiferromagnetic ferrihydrite nanoparticles of two types, bacterial and chemical. The investigations included pulsed switching in fields up to $130 \mathrm{kOe}$ and pulse lengths from 4 to $16 \mathrm{~ms}$. For the samples of two types, we established the following regularities. In the temperature range where the effects related to the irreversible behavior of the magnetization curve are insignificant in quasistatic magnetic fields, the hysteresis effects are observed upon pulsed magnetization switching. The coercivity depends on both parameters determining the external field variation rate, i.e., pulse length and maximum applied field. The first factor is unambiguously related to the relaxation processes characteristic of ferromagnetic nanoparticles: a decrease in the pulse length is equivalent to an increase in the frequency or field variation rate, which results in the coercivity growth. However, the observed increase in the coercivity with increasing maximum applied temperature (up to $\sim 10^{5} \mathrm{Oe}$ ) can be considered to be a feature in the behavior of not only ferrihydrite nanoparticles, but also nanoparticles with an antiferromagnetic order and an uncompensated magnetic moment. According to our data, the similar conclusion can be drawn for antiferromagnetic nickel oxide nanoparticles. This feature should be taken into account when building a theory of the dynamic magnetic hysteresis of antiferromagnetic nanoparticles. In addition, note that a substantial broadening of the temperature range of existence of the magnetic hysteresis in pulsed fields can become a basis for a wider use of materials based on antiferromagnetic nanoparticles.

\section{ACKNOWLEDGMENTS}

The authors thank Yu.V. Knyazev and O.A. Bayukov for the Mössbauer study and M.N. Volochaev for transmission electron microscopy study of the samples. The investigations were carried out on a Hitachi HT7700 transmission electron microscope at the Center for Collective Use of the Krasnoyarsk Scientific Center, Russian Academy of Sciences, Siberian Branch.

This study was supported by the Russian Foundation for Basic Research, the Government of the Krasnoyarsk Territory, and the Krasnoyarsk Territorial Foundation for Support of Scientific and R\&D Activities, project no. 17-42-240138.

\section{REFERENCES}

1. S. Mørup, D. E. Madsen, C. Fradsen, C. R. H. Bahl, and M. F. Hansen, J. Phys.: Condens. Matter 19, 213202 (2007).

2. Yu. L. Raikher and V. I. Stepanov, J. Exp. Theor. Phys. 107, 435 (2008).

3. Yu. L. Raikher and V. I. Stepanov, J. Phys.: Condens. Matter 20, 204120 (2008).

4. Q. A. Pankhurst, N. T. K. Thanh, S. K. Jones, and J. Dobson, J. Phys. D 42, 224001 (2009).

5. K. Dobretsov, S. Stolyar, and A. Lopatin, Acta Otorhinolaryngol. Ital. 35, 97 (2015).

6. S. A. Makhlouf, F. T. Parker, F. E. Spada, and A. E. Berkowitz, J. Appl. Phys. 81, 5561 (1997).

7. S. A. Makhlouf, H. Al-Attar, and R. H. Kodama, Solid State Commun. 145, 1 (2008).

8. C. Diaz-Guerra, M. Vila, and J. Piqueras, Appl. Phys. Lett. 96, 193105 (2010).

9. N. J. O. Silva, V. S. Amaral, A. Urtizberea, R. Bustamante, A. Millán, F. Palacio, E. Kampert, U. Zeitler, S. de Brion, O. Iglesias, and A. Labarta, Phys. Rev. B 84, 104427 (2011).

10. J. F. K. Cooper, A. Ionescu, R. M. Langford, K. R. A. Ziebeck, C. H. W. Barnes, R. Gruar, C. Tighe, J. A. Darr, N. T. K. Thanh, and B. Ouladdiaf, J. Appl. Phys. 114, 083906 (2013).

11. A. E. Bianchi, S. J. Stewart, R. D. Zysler, and G. Punte, J. Appl. Phys. 112, 083904 (2012).

12. D. A. Balaev, A. A. Krasikov, A. A. Dubrovskiy, S. V. Semenov, S. I. Popkov, S. V. Stolyar, R. S. Iskhakov, V. P. Ladygina, and R. N. Yaroslavtsev, Phys. Solid State 58, 287 (2016).

13. D. A. Balaev, A. A. Krasikov, A. A. Dubrovskiy, S. I. Popkov, S. V. Stolyar, R. S. Iskhakov, V. P. Lady- 
gina, and R. N. Yaroslavtsev, J. Appl. Phys. 120, 183903 (2016).

14. N. J. O. Silva, V. S. Amaral, and L. D. Carlos, Phys. Rev. B 71, 184408 (2005).

15. S. A. Makhlouf, F. T. Parker, and A. E. Berkowitz, Phys. Rev. B 55, R14717 (1997).

16. C. Gilles, P. Bonville, H. Rakoto, J. M. Broto, K. K. W. Wong, and S. Mann, J. Magn. Magn. Mater. 241, 430 (2002).

17. N. J. O. Silva, A. Millan, F. Palacio, E. Kampert, U. Zeitler, and V. S. Amaral, Phys. Rev. B 79, 104405 (2009).

18. Yu. L. Raikher, V. I. Stepanov, S. V. Stolyar, V. P. Ladygina, D. A. Balaev, L. A. Ishchenko, and M. Balashov, Phys. Solid State 52, 298 (2010).

19. Ch. Rani and S. D. Tiwari, Phys. B (Amsterdam, Neth.) 513, 58 (2017).

20. D. A. Balaev, S. I. Popkov, A. A. Krasikov, A. D. Balaev, A. A. Dubrovskiy, S. V. Stolyar, R. N. Yaroslavtsev, V. P. Ladygina, and R. S. Iskhakov, Phys. Solid State 59, 1940 (2017).

21. D. A. Balaev, A. A. Dubrovskiy, A. A. Krasikov, S. I. Popkov, A. D. Balaev, K. A. Shaikhutdinov, V. L. Kirillov, and O. N. Mart'yanov, Phys. Solid State 59, 1547 (2017).

22. I. S. Poperechny, Yu. L. Raikher, and V. I. Stepanov, Phys. Rev. B 82, 174423 (2010).

23. I. S. Poperechny and Yu. L. Raikher, Phys. B (Amsterdam, Neth.) 435, 58 (2014).

24. Yu. P. Kalmykov, B. Ouari, and S. V. Titov, J. Appl. Phys. 120, 053901 (2016).

25. R. H. Kodama and A. E. Berkowitz, Phys. Rev. B 59, 6321 (1999).

26. D. A. Balaev, A. A. Krasikov, A. A. Dubrovskii, S. V. Semenov, O. A. Bayukov, S. V. Stolyar, R. S. Iskhakov, V. P. Ladygina, and L. A. Ishchenko, J. Exp. Theor. Phys. 119, 479 (2014).

27. D. A. Balaev, I. S. Poperechny, A. A. Krasikov, K. A. Shaikhutdinov, A. A. Dubrovskiy, S. I. Popkov, A. D. Balaev, S. S. Yakushkin, G. A. Bukhtiyarova, O. N. Mar- tyanov, and Yu. L. Raikher, J. Appl. Phys. 117, 063908 (2015).

28. J. G. E. Harris, J. E. Grimaldi, D. D. Awschalom, A. Chiolero, and D. Loss, Phys. Rev. B 60, 3453 (1999).

29. Chandni Rani and S. D. Tiwari, J. Magn. Magn. Mater. 385, 272 (2015).

30. M. S. Seehra, V. S. Babu, A. Manivannan, and J. W. Lynn, Phys. Rev. B 61, 3513 (2000).

31. D. A. Balaev, A. A. Krasikov, A. A. Dubrovskiy, S. I. Popkov, S. V. Stolyar, O. A. Bayukov, R. S. Iskhakov, V. P. Ladygina, and R. N. Yaroslavtsev, J. Magn. Magn. Mater. 410, 71 (2016).

32. S. V. Stolyar, O. A. Bayukov, Yu. L. Gurevich, V. P. Ladygina, R. S. Iskhakov, and P. P. Pustoshilov, Inorg. Mater. 43, 638 (2007).

33. S. V. Stolyar, R. N. Yaroslavtsev, R. S. Iskhakov, O. A. Bayukov, D. A. Balaev, A. A. Dubrovskii, A. A. Krasikov, V. P. Ladygina, A. M. Vorotynov, and M. N. Volochaev, Phys. Solid State 59, 555 (2017).

34. D. A. Velikanov, Vestn. SibGAU, No. 2 (48), 176 (2013).

35. D. A. Balaev, A. A. Krasikov, S. V. Stolyar, R. S. Iskhakov, V. P. Ladygina, R. N. Yaroslavtsev, O. A. Bayukov, A. M. Vorotynov, M. N. Volochaev, and A. A. Dubrovskii, Phys. Solid State 58, 1782 (2016).

36. S. V. Stolyar, D. A. Balaev, A. A. Krasikov, A. A. Dubrovskiy, R. N. Yaroslavtsev, O. A. Bayukov, M. N. Volochaev, and R. S. Iskhakov, J. Supercond. Nov. Magn. (2017). doi 10.1007/s10948-017-4263-6

37. M. Gich, A. Roig, C. Frontera, E. Molins, J. Sort, M. Popovici, G. Chouteau, D. Martın, Y. Marero, and J. Nogués, J. Appl. Phys. 98, 044307 (2005).

38. A. A. Dubrovskiy, D. A. Balaev, K. A. Shaykhutdinov, O. A. Bayukov, O. N. Pletnev, S. S. Yakushkin, G. M. Bukhtiyarova, and O. N. Martyanov, J. Appl. Phys. 118, 213901 (2015).

Translated by E. Bondareva 